\title{
Phenotypic screening for detection of methicillin-resistant Staphylococcus aureus in clinical settings
}

Methicillin antibiotic was first introduced in 1959. It is not long after its introduction, methicillin-resistant. Methicillinresistant Staphylococcus aureus (MRSA) has been a problem in health care facilities and later in communities [1]. Normally, $S$. aureus is present in the upper respiratory tract, skin, and GI tract [2]. With unfavorable conditions, the colonized flora can be pathogenic [2]. These conditions occur mainly in immunocompromised hosts such as cancer, connective tissue diseases, organ transplantation, chronic diseases, newborn facilities, and nursing homes [3]. However, some patients who develop MRSA infection do not have any documented risk factors.

Through horizontal gene transfer, $S$. aureus develops a resistance to methicillin and becomes MRSA. MRSA acquires multiple drug resistance to beta-lactam antibiotics including penicillin, methicillin, oxacillin, and cephems such as cephalosporins. MRSA has mec gene ( $m e c A$, or a related variant known as mec $($ ), which possesses a structural component capable of encoding penicillin-binding protein 2a (PBP2a) that establishes the resistance to methicillin and other semisynthetic penicillinase-resistant beta-lactams [4, 5]. Methicillin-sensitive $S$. aureus or MSSA does not have mec gene.

MRSA can cause a wide range of infections such as endocarditis, septicemia, osteomyelitis, and soft tissue infections [6]. They could start with nasal colonization at hospital admission and subsequently became widespread and could be life-threatening [7]. Therefore, it is essential that providers identify MRSA early so that prompt and timely treatment can be given to achieve desirable outcomes. Traditional methods used to process surveillance cultures take $48-72 \mathrm{~h}$ to yield results. However, newer techniques shorten the amount of time required to detect MRSA in surveillance cultures. There are a variety of chromogenic agars available that can detect MRSA stains within $24 \mathrm{~h}$. One of these chromogenic selective agars contains cefoxitin and detects a majority of MRSA isolates within $24 \mathrm{~h}$, while commercially available real-time PCR tests for mecA can detect MRSA within $2 \mathrm{~h}$ [8].

The gold standard for the detection of MRSA is PCR of the mecA gene and the latex agglutination test for the detection of penicillin-binding protein 2a (PBP2a) $[9,10]$. However, the PCR of the mecA gene may not be available or maybe too costly for a large number of laboratories in developing countries to use as a routine method. The diagnosis of MRSA has to resort to the phenotypic identification method for MRSA.

The phenotypic methods commonly used for MRSA identification include oxacillin MIC (agar dilution/broth dilution) or E-strip, oxacillin disc diffusion, oxacillin agar screening plates, and the cefoxitin disc diffusion methods. Longanathan A et al. in this issue [11], report an evaluation of various phenotypic methods with genotypic screening for the detection of MRSA [5]. They suggest that in routine disc diffusion tests, oxacillin can be replaced by cefoxitin for the detection of MRSA and that if PCR is too costly, many laboratories can routinely resort to the combination of simple phenotypic methods, such as cefoxitin disc diffusion and oxacillin MIC, for the detection of MRSA.

Clinicians may have to balance the test techniques available, given the seriousness of the problems of the patients at hand as well as the resources available at their disposal. Efforts to prevent $S$. aureus transmission through the systematic decolonization of facilities can also be some effective measures [12].

*Correspondence to: Editorial Office of Asian Biomedicine, Faculty of Medicine, Chulalongkorn University, Bangkok 10330, Thailand, e-mail:abmjournal@chula.ac.th

D Open Access. ๑ 2019 Editorial Office of Asian Biomedicine, published by Sciendo. (๔) BY-NC-ND This work is licensed under the Creative Commons Attribution NonCommercial-NoDerivatives 4.0 License. 


\section{References}

[1] Benner EJ, Kayser FH. Growing clinical significance of methcillinresistant Staphylococcus aureus. Lancet. 1968; 2:741.

[2] Bradley SF. MRSA colonisation (eradicating colonisation in people without active/invasive infection). BMJ Clin Evidence. 2011; 2011:923.

[3] Boyce JM. Methicillin-resistant Staphylococcus aureus in hospitals and long-term care facilities: microbiology, epidemiology, and preventive measures. Infect Control Hosp Epidemiol. 1992; 13:725-37.

[4] Musser JM1, Kapur V. Clonal analysis of methicillin-resistant Staphylococcus aureus strains from intercontinental sources: association of the mec gene with divergent phylogenetic lineages implies dissemination by horizontal transfer and recombination. J Clin Microbiol. 1992; 30:2058-63.

[5] Kriegeskorte A, Idelevich EA, Schlattmann A, Layer F, Strommenger $\mathrm{B}$, Denis O, et al. Comparison of different phenotypic approaches to screen and detect mecC-harboring methicillin-resistant Staphylococcus aureus. J Clin Microbiol. 2017; 56:e00826-17.

[6] Simpson S. Methicillin resistant Staphylococcus aureus and its implications for nursing practice: a literature review. Nurs Pract. 1992; 5:2-7.
[7] Chambers HF. Treatment of infection and colonization caused by methicillin-resistant Staphylococcus aureus. Infect Control Hosp Epidemiol. 1991; 12:29-35.

[8] Warren DK, Liao RS, Merz LR, Eveland M, Dunne WM Jr. Detection of methicillin-resistant Staphylococcus aureus directly from nasal swab specimens by a real-time PCR assay. J Clin Microbiol. 2004; 42:5578.

[9] Luteijn JM, Hubben GA, Pechlivanoglou P, Bonten MJ, Postma MJ. Diagnostic accuracy of culture-based and PCR-based detection tests for methicillin-resistant Staphylococcus aureus: a meta-analysis. Clin Microbiol Infect. 2011; 17:146-54.

[10] Wanger AR, Moore DG, LaRocco MT. Latex agglutination for rapid identification of methicillin-resistant Staphylococcus aureus recovered from selective media. Eur J Clin Microbiol Infect Dis. 1991; 10:564-7.

[11] Laganathan A, Manohar P, Eniyan K, Jayaraj R, Nachimuthu R. Evaluation of various phenotypic methods with genotypic screening for detection of methicillin-resistant Staphylococcus aureus. Asian Biomed (Res Rev News). 2019; 13:225-33.

[12] Popoola VO, Milstone AM. Decolonization to prevent Staphylococcus aureus transmission and infections in the neonatal intensive care unit. J Perinatol. 2014; 34:805. 